\title{
Experimenting with multistressors
}

Understanding climate change impacts on species and ecosystems is complex, as individual systems will be exposed to different effects. Ocean acidification is one example stressor (or driver), but it is unlikely to occur in isolation; climate change will result in multiple stressors to organisms, such as warming, nutrient changes and stratification of the water column. All of these will affect and interact with each other in different ways. To date, most research has considered a single stressor. To understand what the future ocean may look like, there is a need to consider many possible permutations of stressors, and their synergies and antagonistic effects.

This is not a simple task and whilst there have been calls to consider interaction (K. J. Kroeker et al. Biol. Lett. 13, 20160802; 2017) it is a relatively new area of research. To help facilitate researchers getting up and running with the right protocols in their marine research, a new online resource is launching. Philip Boyd, of the Institute for Marine and Antarctic Studies at the University of Tasmania, Australia, speaking on behalf of the Scientific Committee on Oceanic Research (SCOR) Working Group 149 (WG149), outlines the Best Practice Guide for Multiple Drivers Marine Research (Boyd, P. W. et al. https://doi. org/10.25959/5c92fdf0d3c7a; 2019). The guide includes a web-based simulation tool for experimental design, the Multiple Environmental Driver Design Lab for Experiments (MEDDLE; www.meddlescor149.org). "MEDDLE is designed to allow individuals to tailor - it allows different permutations of stressors, different treatment levels and different organisms to address the question being asked," Boyd explains.

The idea was sparked at the 4 th International Symposium on the Ocean in a High- $\mathrm{CO}_{2}$ World meeting in Hobart, Australia, back in 2016, during a 50-person workshop that included around 30 earlycareer researchers. "There was push back on the ocean acidification guide, which is a book with over 250 pages. If that is what was required for an individual driver, what would be needed for multiple ones?" Boyd says. A more flexible and nimble

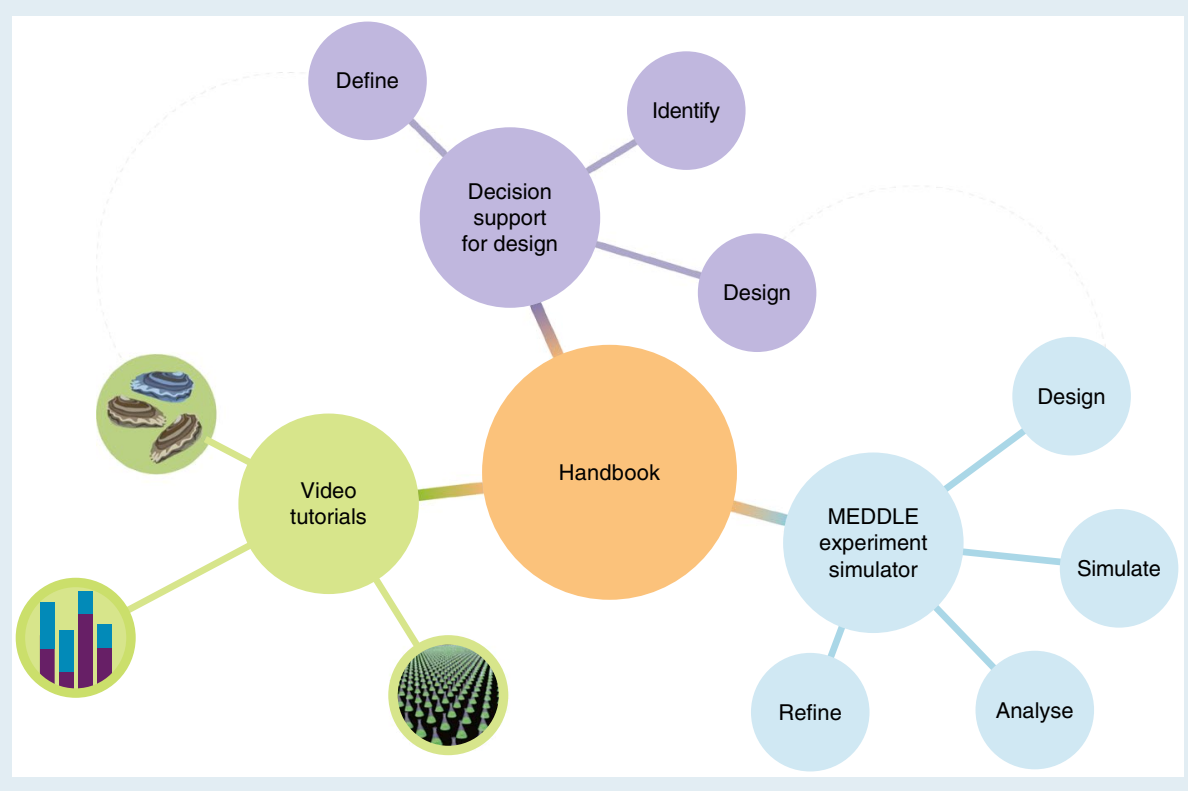

Credit: Philip Boyd

approach was needed to accommodate the complexities of designing experiments involving multiple stressors. This challenge was taken up by SCOR WG149, a 20-person working group, to develop a new approach.

The Best Practice Guide has been created to ensure robust experimental work that is comparable and reproducible, and should generate meaningful results that can feed into the IPCC reports. The guide provides different types of learning, starting with a series of short introduction videos on multistressor work, which are complemented by themed videos with advice to enable the user to identify relevant stressors and design their experiment. The Decision Support Tool builds on this by taking the user further into the planning process via a threestep iterative loop to define the research question; identify the responses, stressors/ drivers and design; and finalise the design. Within MEDDLE there is the opportunity for the user to take control - inputting the experiment design created in the Decision Support Tool, as well as moving sliders to manipulate variables, allowing interactions in different ways - and the tool suggests appropriate statistical tests. This simulation can include up to three variables and tests the experimental design, hoping to optimize the design and minimize lost hours in the laboratory.

The international team have endeavoured to make the new resource user-friendly, and the video transcripts will be made available in 8 languages: English, Chinese, French, German, Japanese, Portuguese, Spanish and Swedish. SCOR WG149 are excited to be launching this tool, which they hope will support those new to this area of research as well as those more established in the community, with different entry points to accomodate all levels of experience. These tools will not be static, as it is anticipated that they will grow based on feedback and as greater understanding of the field is generated.

Understanding multistressors in the marine environment is necessary to ensure future ocean health, and this tool looks set to support researchers in gaining that knowledge.

\section{Bronwyn Wake}

Published online: 26 April 2019

https://doi.org/10.1038/s41558-019-0475-Z 